\title{
Study of Prognostic Factors of Acute Pancreatitis in A Teaching Hospital
}

\author{
MD. ABDUL AHAD ${ }^{1}$, KAMAL UDDIN AHMED $^{2}$, SM KAMAL $^{3}$, ARM SAIFUDDIN EKRAM $^{4}$
}

\begin{abstract}
This hospital based cross sectional descriptive study was performed to find out the prognostic factors of acute pancreatitis from the available clinical, biochemical, haematological and radiological data. A total of 50 cases were studied. Peak incidence was in the fourth decade. Among the known aetiological factors $18 \%$ were due to gallstone disease, $10 \%$ alcoholism, $4 \%$ pancreatobiliary ascariasis, but $60 \%$ were idiopathic. In this study $82 \%$ patients were had mild and $18 \%$ had severe acute pancreatitis. Overall mortality rate was $6 \%$, mortality was significantly higher $(33.33 \%)$ in severe acute pancreatitis (33.3\% vs $0 \%$ ). A higher mortality was associated with concomitant medical or surgical diseases, leucocytosis. Other factors associated with a higher mortality were high blood glucose, serum creatinine level and a lower serum albumin and calcium level. This study highlights the need of further study with more detailed recording of relevant data from primary care hospital to find out the clinical pattern of complications and prognostic factors in our country.
\end{abstract}

Keyword: Acute Pancreatitis, Prognosis, Mortality

\section{Introduction:}

Acute pancreatitis is an acute inflammatory process of the pancreas with variable involvement of regional tissues and remote organ systems. ${ }^{1}$ The incidence of pancreatitis varies in different countries and depends on cause, e.g., alcohol, gallstones, metabolic factors and viral infections. The estimated incidence in industrialized countries is 10-20/ 100,000 per year. ${ }^{2}$ There are many causes of acute pancreatitis, but the mechanisms by which these conditions trigger pancreatic inflammation have not been identified. Approximately 2 to $5 \%$ of cases of acute pancreatitis are drug-related. Drugs cause pancreatitis either by a hypersensitivity reaction or by the generation of a toxic metabolites. $^{3}$

Early accurate diagnosis is very important for its management. Symptoms of acute pancreatitis vary considerably. Some patients have mild epigastric discomfort that may mimic peptic ulcer. Others suffer from an acute catastrophic illness that cannot be readily distinguished from such severe intraabdominal condition as perforated duodenal ulcer or mesenteric infarction. For this reason the clinician must carefully evaluate information derived from other sources that supplement the history and physical examination including laboratory tests, imaging studies before arriving at a correct diagnosis of acute pancreatitis. Removal of factors that may have precipitated the attack is one of the important aspects of management of acute pancreatitis. If the cause of the attack can be eliminated there will be no further attacks and the pancreas will return to normal in terms of its morphology and function. ${ }^{4}$ The variation in the etiology of pancreatitis from series to series is quite marked and depend on the country of origin. ${ }^{5}$ In this potentially fatal disease, mortality depends on the severity. Most attacks of acute pancreatitis are mild and death is unusual in such patients. Mortality and morbidity is very high in those with severe acute pancreatitis. So grading of severity is of critical importance in providing appropriate therapy in acute pancreatitis. Studies suggest that outcome of an attack of acute pancreatitis can be improved by early treatment and therefore, urgent treatment of severe acute pancreatitis will reduce morbidity and mortality. ${ }^{6}$

Many attempts have been made to improve the recognition of a severe attack at the onset. Combinations of clinical and laboratory data that can indicate the severity of acute pancreatitis within first 48 hours after admission have been developed by Ranson and colleagues and clinician from Glasgow. APACHE II illness grading system can also be used to predict severity. ${ }^{7}$ All those systems are cumbersome and such combination system has low sensitivity in clinical practice. ${ }^{8} \mathrm{~A}$ number of risk factors have been evaluated in

1. Associate Professor, Dept.of Gastroenterology, Khulna Medical College.

2. Junior Consultant Medicine, General Hospital, Faridpur.

3. Associate Professor, Dept. of Medicine, Khulna Medical College.

4. Professor, Dept. of Medicine, Rajshahi Medical College.

Correspondence: Dr. Md. Abdul Ahad, Associate Professor, Department of Gastroenterology, Khulna Medical College. 
the clinical setting. These include markers of pancreatic injury and of inflammatory response, together with simple routine tests and imaging methods. ${ }^{9}$ However, these markers are not available in every hospital and some of them proved not to be useful in current clinical practice. The main objective of this study was to observe the prognostic factors of acute pancreatitis.

\section{Material and Methods:}

This was a cross sectional descriptive study carried out at Department of Medicine and Department of Surgery, Rajshahi Medical College Hospital from January 2006 to April 2007. In this study a total 50 cases of acute pancreatitis were analyzed. Patients were selected using the inclusion criteria of appropriate clinical evidence, associated with an elevation of serum amylase and/or urinary amylase irrespective of age and sex. Normal value of serum amylase was accepted upto $220 \mathrm{U} / \mathrm{L}$ and that of urinary amylase was accepted upto 400 U/L. Exclusion criteria were a serum amylase level greater than upper limit of normal value with a diagnosis of pancreatic or periampulary cancer, chronic pancreatitis, perforation of the gut, and diabetic ketoacidosis.

A predesigned case record form was used and detailed clinical history was recorded from the patient's history or written history sheet. Age, sex, address, symptoms and signs were noted. Relevant past history, family history and personal history, especially alcohol consumption were recorded. Associated medical diseases like diabetes mellitus, hypertension, chronic renal failure, bronchial asthma, chronic obstructive pulmonary disease (COPD) and ischemic heart disease were also noted. The most likely aetiological factor was identified by analyzing history, physical examination and relevant investigations. Gall stone related acute pancreatitis was based upon the identification of stone in the gall bladder or in the common bile duct (CBD) by sonography. Alcohol, mumps or trauma was considered to be aetiological factor from the history. A pancreatobiliary ascariasis was diagnosed on the basis of sonographic findings. When the triglyceride level was above $880 \mathrm{mg} / \mathrm{dl}$, it was considered to be the aetiological factor. When no known cause was found it was termed idiopathic.

Criteria of severity were based on the Glasgow criteria. To evaluate prognostic factors, a standard scoring system like Ranson, Glasgow or APACHE-II, all data were not available. Available data of all the studied patients were analysed to findout the important prognostic factors. Data of survivors and non-survivors were compared-two demographic (age and sex), two haematological (haemoglobin and total white cell count), five biochemical (blood sugar, serum bilirubin, aspertate aminotransferase, serum albumin and serum calcium) parameters. Blood glucose level in those patients previously diagnosed diabetic were not analyzed for the prognostic purpose. Patients were stratified in four age groups (i.e., below 20 years, 20 to 40 years, 41 to 60 years and above 60 years).Survivors were defined as patients discharged alive from the hospital and non-survivors as those who died from acute pancreatitis or its complications during hospitalization. Approval was taken from the ethical committee.

\section{Results}

Of the 50 patients studied, thirty-two patients were male and eighteen were female. Age range was 13 to 74 years, with a mean age of 37 years. Out of 50 patients, $9(18 \%)$ had gallstone related pancreatitis. Pancreatobiliary ascariasis was found in 2 (4\%) patients. Alcoholism was found in 5 (10\%) cases, trauma in 1 (2\%) case, mumps in 2 (4\%), hypertriglyceridemia in 1 (2\%) cases. No cause was found in 30 (60\%) cases

Table-I

Ultrasonographic findings of acute pancreatitis $(N=50)$

\begin{tabular}{lcccccc}
\hline \multirow{2}{*}{ USG Findings } & \multicolumn{2}{c}{ Male } & & \multicolumn{2}{c}{ Female } \\
\cline { 2 - 3 } \cline { 6 - 7 } Swollen/ Enlarged Pancreas & 18 & $56.25 \%$ & & 5 & $27.77 \%$ \\
Normal Pancreas & 10 & $31 \%$ & & 13 & $72 \%$ \\
Cholelithiasis & 1 & $3 \%$ & & 4 & $22.22 \%$ \\
Choledocholithiasis & 1 & $3 \%$ & & 2 & $11 \%$ \\
Ascites & 4 & $12.50 \%$ & & 2 & $11.11 \%$ \\
Biliary Ascariasis & 1 & $3 \%$ & & 4 & $22 \%$ \\
\hline
\end{tabular}

In Table-I, Snographic evaluation of patients are showed which revealed 23 (46\%) patients had swollen or enlarged pancreas. Cholelithiasis were identified in $9(18 \%)$ patients, choledocholithiasis in 3 (6\%) patients, biliary ascariasis in 2 (4\%) patients. Pancreas sonologically appeared normal in 23 (46\%) patients.

Table-II

Hematological profile of acute pancreatitis $(N=50)$

\begin{tabular}{lcccccc}
\hline & \multicolumn{2}{c}{ Male } & & \multicolumn{2}{c}{ Female } \\
\cline { 2 - 3 } \cline { 6 - 7 } Complication & Total & Percent & & Total & Percent \\
\hline $\mathrm{Hb} \% \geq 10 \mathrm{gm} / \mathrm{dl}$ & 28 & $88 \%$ & & 15 & $84 \%$ \\
$\mathrm{Hb} \% \leq 10 \mathrm{gm} / \mathrm{dl}$ & 4 & $12 \%$ & & 3 & $16 \%$ \\
$\mathrm{TC}$ of $\mathrm{WBC} \geq 15,000 / \mathrm{mm} 3$ & 12 & $38 \%$ & & 3 & $16 \%$ \\
$\mathrm{TC}$ of $\mathrm{WBC} \leq 15,000 / \mathrm{mm} 3$ & 20 & $62 \%$ & & 15 & $84 \%$ \\
\hline
\end{tabular}


Of the two heamatological parameters tested, haemoglobin level was10 $\mathrm{gm} / \mathrm{dl}$ or above were 43 (86\%) patients and 7 (14\%) patients were below this level. TC of WBC $\geq 15000 /$ $\mathrm{mm}^{3}$ was in $15(30 \%)$ patients but 35 patients were below this level. Out of two haematological parameters tested a higher leucocyte count was associated with high mortality, but a low haemoglobin level was not associated with increased mortality (Table-II).

Table-III

Bio-Chemical profile of acute pancreatitis $(N=50)$

\begin{tabular}{lcccccc}
\hline & \multicolumn{2}{c}{ Male } & & \multicolumn{2}{c}{ Female } \\
\cline { 2 - 3 } \cline { 6 - 7 } Parameters & Total & Percent & & Total & Percent \\
\hline RBS, $(\geq 10 \mathrm{mmol} / \mathrm{L})$ & 3 & $9 \%$ & & 0 & $0 \%$ \\
RBS, $(\leq 10 \mathrm{mmol} / \mathrm{L})$ & 29 & $91 \%$ & & 18 & $100 \%$ \\
S.Bilirubin $(\geq 1.2 \mathrm{mg} / \mathrm{dl})$ & 1 & $3 \%$ & & 1 & $5 \%$ \\
S.Bilirubin $(\leq 1.2 \mathrm{mg} / \mathrm{dl})$ & 31 & $97 \%$ & & 17 & $95 \%$ \\
S. ALT $(\geq 45 \mathrm{IU} / \mathrm{L})$ & 2 & $6 \%$ & & 1 & $5 \%$ \\
S. ALT $(\leq 45 \mathrm{IU} / \mathrm{L})$ & 30 & $94 \%$ & & 17 & $95 \%$ \\
S. Albumin $(\geq 3.2 \mathrm{gm} / \mathrm{dl})$ & 30 & $94 \%$ & & 16 & $89 \%$ \\
S. Albumin $(\leq 3.2 \mathrm{gm} / \mathrm{dl})$ & 2 & $6 \%$ & & 2 & $11 \%$ \\
S. Calcium $(\geq 8.5 \mathrm{mg} / \mathrm{dl})$ & 31 & $97 \%$ & & 15 & $84 \%$ \\
S. Calcium $(\leq 8.5 \mathrm{mg} / \mathrm{dl})$ & 1 & $3 \%$ & & 3 & $16 \%$ \\
\hline
\end{tabular}

Table III showing low serum calcium and albumin and a high blood glucose level were associated with higher mortality. Serum bilirubin and aspartate aminotransferase were found not to associated with increased mortality.

Table-IV

Complication Profile of acute pancreatitis $(N=50)$

\begin{tabular}{lccccc}
\hline \multirow{2}{*}{ Complication } & \multicolumn{2}{c}{ Male } & & \multicolumn{2}{c}{ Female } \\
\cline { 2 - 3 } \cline { 6 - 7 } Hyperglycemia & Total & Percent & & Total & Percent \\
\hline Hypocalcemia & 0 & $0 \%$ & & 0 & $0 \%$ \\
Pseudocyst & 1 & $3 \%$ & & 3 & $17 \%$ \\
Ascites with Pl.effusion. & 2 & $6 \%$ & & 6 & $33 \%$ \\
Effusion & 3 & $9 \%$ & & 0 & $0 \%$ \\
Upper GI Bleeding & 1 & $3 \%$ & & 1 & $5 \%$ \\
Obstructive Jaundice & 0 & $0 \%$ & & 3 & $17 \%$ \\
\hline
\end{tabular}

Only 20 (40\%) patients developed complications. Out of these 20 patients, 7 patients were male and 13 patients were female. Hypocalcaemia developed in 4 (8\%) patients, pancreatic pseudocyst in 3 (6\%) patients, ascites with left sided pleural effusion in 8 (16\%) patients, upper gastrointestinal bleeding in 2(4\%) patients and obstructive jaundice in 3 (6\%) cases ( Table-IV).

Table-V

Prognostic profile of acute pancreatitis (Male=32), (Female-18)

\begin{tabular}{lcccccc}
\hline \multicolumn{7}{c}{ Male } \\
\hline Prognosis & $<20$ & $21-40$ & $41-60$ & $>60$ & Total & percentage \\
Survivor & 1 & 17 & 9 & 3 & 30 & $93.75 \%$ \\
Non-Survivor & 1 & 1 & 2 & $6.25 \%$ \\
\hline \multicolumn{7}{c}{ Female } \\
\hline Prognosis & $<20$ & $21-40$ & $41-60$ & $>60$ & Total & percentage \\
Survivor & 4 & 8 & 4 & 1 & 17 & $94.44 \%$ \\
Non-Survivor & & 1 & & & 1 & $5.56 \%$ \\
\hline
\end{tabular}

Out of 50 patients, 41 (82\%) were graded as mild and 9 (18\%) as severe. A total 3 (6\%) patient died. A significantly higher mortality was found in patients with severe pancreatitis, than those with mild attack ( $0 \%)$. Mortality was related to the presence of fever (temp $>100^{\circ} \mathrm{F}$ ) at admission and presence of concomitant medical diseases (Table-V).

\section{Discussion}

It was retrospective study on 50 cases of acute pancreatitis. Only those cases who were admitted into RMCH were subjected to study. Acute pancreatitis is a disease that varies in severity ranging from a mild and self limited illness to a very severe and rapidly progressive condition leading to multiple organ failure and eventually to death. An early diagnosis and identification of those who are at risk of development of severe disease and rapid institution of therapy might reduce in morbidity and mortality. If the aetiological factor/factors are known and can be eliminated, further attacks can also be prevented. In this study the age range of patient was 13 to 74 years with a peak incidence is in the fourth decade. In western countries the peak incidence of alcoholic pancreatitis is in the fifth decade, whereas pancreatitis associated with gallstones and other cases peaks in the seventh decade. ${ }^{2}$ In our country the lower age incidence of acute pancreatitis may be due to lower life expectancy of Bangladeshi people, occurrence of gallstone disease in the female in early age due to early and multiple pregnancy. Study data showed that ascariasis, mumps and trauma are common aetiological factors, that more commonly affect younger age group. In industrialized countries acute pancreatitis more commonly affects male than female and this is due to increased alcohol consumption by male. ${ }^{2}$ In 
female it is more commonly related to gallstone disease. In this study there is male predominance (Male: Female $=1.78: 1$ ).

In different series of the USA, Western Europe and Asia, gallstones are the most common cause of acute pancreatitis accounting for approximately $45 \%$ cases. Alcohol is the second most common cause. However, the variation of aetiology of pancreatitis is quite marked and depend on country of origin. ${ }^{8}$ In this study most cases (60\%) were idiopathic i.e. no cause is found. These cases are probably related to viral or bacterial infection which needs further exploration. Gallstone ranks the second among the known aetiological factors (18\% cases). This high incidence of idiopathic acute pancreatitis in our country is due to higher prevalence of infections because of poor nutrition, poor sanitation, over crowding and lack of knowledge. In our series only $10 \%$ cases were due to alcohol. This low incidence in contrast to Western countries reflects the socio-cultural and religious factors that discourage alcohol consumption in our country. Mumps is a rare cause of acute pancreatitis in developed countries. ${ }^{10}$ But in our study it seems to be an important aethological factors accounting for $4 \%$ cases. This higher incidence may due to over crowding. Blunt trauma to the abdomen is a recognized cause of acute pancreatitis, which results from the disruption of the pancreatic ductal system. Blunt trauma from road traffic accidents is the dominant cause in Europe while direct injury by a knife or bullet is more common in North America. ${ }^{11}$ In this series there were only $1(2 \%)$ case which was caused by blunt trauma to abdomen. Hypertriglyceridemia was responsible in 1(2\%) case. Triglyceridemia levels exceeding $\geq 880 \mathrm{mg} / \mathrm{dl}$ deciliter increases the risk of pancreatitis. In this study 1 (2\%) case developed pancreatitis due to hypertriclyceridemia which is consistent with that of other countries. ${ }^{12}$

In this study a large group of patient, no cause could be identified. This so called idiopathic cause is the most common cause of acute pancreatitis in most Western series. Recently, two prospective studies on consecutive patients with apparently idiopathic pancreatitis showed that two-thirds to three-fourths had microlithiasis as the presumed cause, as documented by biliary drainage studies, follow-up sonogram and ERCP with sphinterotomy or cholecytectomy. 13 No such data was available in the hospital documents of studied patients in this series. All patients of this series under went ultrasonographic examinations. More than 46\% of patients had sonographic abnormalities of pancreas. Fourty-six (46\%) percent pancreas appeared to be normal which may be due to poor visualization of the gland. Despite remarkable advances in the understanding of pathophysiology of acute pancreatitis and advances in supportive and intensive care, the overall mortality rate for patients with acute pancreatitis has remained in the range of $10 \%$ for over last 40 yrs. ${ }^{11}$ A large prospective study showed that 3 of every 4 patients with acute pancreatitis will have a mild form of the disease which promptly responds to supportive measure. The fourth patient, however, will have a severe episode and approximately half of those patients will suffer from a complication and one third of them will die. $^{14}$

In our series, $41(82 \%)$ patients had mild and 9 (18\%) had severe acute pancreatitis with an overall mortality rate of $6 \%$. Now-a-days infectious complications of acute pancreatitis account for more than $80 \%$ of all death from this disease. ${ }^{15}$ It seems clear, that if the mortality rate in acute pancreatitis is to be reduced, attention must be directed toward the identification and management of patients with various complications of infections. Early identification of these patients at increased risk for the development of complications may have significant therapeutic implications. Careful and repeated clinical evaluation is clearly of paramount importance in the management of individual patients. These include age, fever, physical abnormality of the chest, a palpable mass and detectable intra abdominal fluid. Increased age is associated with a bad prognosis has been widely recognized and simply reflect a decreased physical reserve in the older population. In Ranson's experience the mean age of patients who died and of those who had a complicated clinical course were significantly higher than those who recovered uneventfully. ${ }^{16}$ Fan et al. have observed a mortality rate of $21.3 \%$ in patients over 75 yrs old and $5.9 \%$ in the younger population. ${ }^{13}$ The high mortality was accounted for by a higher incidence of death related to concomitant medical or surgical diseases in the same hospital admission rather than to complications resulting directly from the pathological process of acute pancreatitis. When only deaths due to complications of acute pancreatitis were analyzed, the mortality rate was not significantly different between the young and elderly groups. The reported relationship between patients sex and prognosis for acute pancreatitis varies. No relationship between the sex of the patients and prognosis has been demonstrated by Ranson and Brisando and our result also supported it. ${ }^{11,17}$

The overall white cell count may reflect the severity of the inflammation and a relationship between the degree of early leucocytosis and the severity of the pancreatitis has been noted by many authors. ${ }^{18}$ In this study, a higher total white cell count was associated with higher mortality and among the biochemical parameters tested a high blood sugar and 
serum creatinine level and a lower serum albumin and calcium levels were significantly associated with a higher mortality. However, this study did not show any relationship between serum bilirubin, aspartate transaminase (AST), alkaline phosphatase, serum potassium levels and a higher mortality. A higher aspartate transaminase (AST) and hypokalaemia were not found to be associated with a higher mortality rate. But in Ranson's and Brisando's experience a high blood sugar, serum creatinine and asparatate transaminase and low serum calcium and albumin were associated with poor prognosis. 11,17

Concomitant medical diseases like diabetes mellitus, bronchial asthma, COPD, CRF and fever $\geq 100^{\circ} \mathrm{F}$ within two days of onset of abdominal pain should be considered as poor prognostic factors, similarly a white blood cell count $\geq 15,000 / \mathrm{mm}^{3}$, a high serum creatinine and a low serum albumin, calcium and presence of pleural effusion should also be considered as poor prognostic factors and such patients should be in managed in a high dependency or intensive care unit.

\section{Conclusion}

Acute pancreatitis is a neglected disease in our country. We are not aware of the actual scenario regarding the incidence, prevalence, cause or mortality rate of this treatable disease. Early recognition of bad prognostic factors can result in appropriate and timely management of acute pancreatitis.

\section{Conflict of Interest: None}

\section{References:}

1. Palmer K R, Penman I D, Paterson Brown S. Alimentary Tract And Pancreatic Disease.In: Boon N A, Colledge NR, Walker BR, Hunter AA editors. Davidson's Principles and Practice of Medicine. Churchill Livingstone; 20th edition. 2006: 904-909

2. Sargen K, Kingsnorth A. Management of Gallstone Pancreatitis: Effects of Deviation from Clinical Guidelines. J Pancreas (Online) 2001; (5):317-322

3. Norton J, Berger G, Philip P. Acute and Chronic pancreatitis; Harrison's Principles of Internal Medicine. McGraw Hill. 20 $0^{\text {th }}$ edition 2005: 1895-1906

4. Banks PA. Acute and Chronic Pancreatitis. In: Fieldman and Sleisenger (editors). Sleiscnger and Fordtran's Gastrointestinal and Liver disease. $6^{\text {th }}$ edition. Vol 1. Philadelphia: WB Saunders Co, 1998: 809-57
5. Wyncell DL. The management of severe acute necrotizing pancreatitis: an evidence-based review of the literature. Intensive Care Med 1999; 25: 146

6. McMahon MJ. Acute pancreatitis. In: Misiewics JJ, Pounder RE, Veneable CW, editors. Diseases of the Gut and Pancreas. $2^{\text {nd }}$ edition. London: Blackwell Scientific Publication. 1994; 427-38

7. Lece T, Shaw D. Comparison of three Glasgow multifactor prognostic scoring systems in acute pancreatitis. Br J Surg 1988; 75:460-462

8 Steinberg WT. Acute pancreatitis. N Engl J Med 1994; 330 : 1198

9. Dervenis C, Bassi C. Evidence-based assessment of severity and management of acute pancreatitis. Br J Surg 2000; 87: $257-258$

10. M. Economou, M. Zissis. Infectious cases of acute pancreatitis. Annals of Gastroenterology 2000;13(2):98-101

11. Ranson JHC. Prognostic signs and the role of operative management in acute pancreatitis. Surg Gynocl Obstet 1994; 139: 69-81

12. Field M J, Burnett L, Sullivan D.R., Stewart P. Clinical Biochemistry and Metabolism. In: Boon N A, Colledge NR, Walker BR, Hunter A A editors. Davidson's Principles and Practice of Medicine. Churchill Livingstone; 20th edition. 2006: p. 451

13. Fan ST, Lai EC, Mok FP. Early treatment of acute biliary pancreatitis by endoscopic papillotomy. N Engl JMed 1993; 328: 228

14. Kanus WA, Draper EA, Wagner DP, Zimmerman JE. APPACHE II: A severity of disease classification system. Crit Care Med 1985; 13: 18-829

15. Beger HG, Bittner R, Silvia Block, Bqchler M. Bacterial contamination of pancreatic necrosis: a prospective clinical study. Gastroenterology 1986; 91:433- 8

16. Lece T, Shaw D. Comparison of three Glasgow multifactor prognostic scoring systems in acute pancreatitis. Br J Surg 1988; 75:460-463

17. Brisando G, Maria G, Ferrante A, Civello IM. Evaluation of prognostic factors in patients with acute pancreatitis. HepaGastroenterol 1999; 46: 1990-1997

18. Norman J. The Role of cytokines in the pathogenesis of acute pancreatitis. Am J surg 1998; 175: 76 